\title{
Design optimization based on state problem functionals
}

\author{
Anders Klarbring \\ Linköping University Post Print
}

Tweet

N.B.: When citing this work, cite the original article.

The original publication is available at www.springerlink.com:

Anders Klarbring, Design optimization based on state problem functionals, 2015, Structural and multidisciplinary optimization (Print), (52), 2, 417-425.

http://dx.doi.org/10.1007/s00158-015-1240-1

Copyright: Springer Verlag (Germany)

http://www.springerlink.com/?MUD=MP

Postprint available at: Linköping University Electronic Press

http://urn.kb.se/resolve?urn=urn:nbn:se:liu:diva-120336 


\title{
Design optimization based on state problem functionals
}

\author{
Anders Klarbring \\ Division of Solid Mechanics \\ Department of Management and Engineering \\ The Institute of Technology \\ Linköping University \\ SE-581 83 Linköping, Sweden \\ E-mail: anders.klarbring@liu.se
}

\begin{abstract}
This paper presents a general mathematical structure for design optimization problems, where state problem functionals are used as design objectives. It extends to design optimization the general model of physical theories pioneered by Tonti [38, 39] and Oden and Reddy [31, 32]. It turns out that the classical structural optimization problem of compliance minimization is a member of the treated general class of problems. Other particular examples, discussed in the paper, are related to Darcy-Stokes flow and pipe flow models. A main novel feature of the paper is the unification of seemingly different design problems, but the general mathematical structure also explains some previously not fully understood phenomena. For instance, the self-penalization property of Stokes flow design optimization receives an explanation in terms of minimization of a concave function over a convex set.
\end{abstract}

\section{Introduction}

A frequent design objective in structural optimization, particularly in topology optimization, is the so-called compliance. For a discrete structure where external prescribed forces are represented by a vector $f$ and where the corresponding displacement vector is $u$, compliance is defined as

$$
\text { Compliance }=\frac{1}{2} f^{T} u .
$$

The reason that compliance has become such a frequently used design objective is a mixture consisting of engineering relevance, physical appeal and mathematical convenience. An essential feature, however, is that an optimal structure in the sense of minimum compliance, often has certain uniform strength or stress properties. For instance, minimizing compliance for a truss under a volume constraint by varying cross-sectional areas of bars, will result in uniform stresses, and this problem can be shown [22] to be equivalent to the problem of minimizing weight under strength or stress constraints. Also frequently referred to and 
used is the property that compliance is equivalent to total strain energy (hence the factor 1/2). However, as pointed out in Klarbring and Strömberg [27], a more useful equivalence than the one between compliance and strain energy is that between compliance and (negative) equilibrium potential energy. Mathematically, these equivalences can be summarized as

$$
\frac{1}{2} f^{T} u=\frac{1}{2} u^{T} K u=-\min _{v} \Pi(v)
$$

where $K$ is the stiffness matrix and the potential energy for any kinematically admissible displacement state $v$ is

$$
\Pi(v)=\frac{1}{2} v^{T} K v-f^{T} v
$$

The last equality of (1) hints at a saddle point property of stiffness optimization, clarifies how non-zero prescribed displacements should be treated and implies that sensitivities follow from a theorem of Clarke [12] (see also Corollary 4.4.5 in [23]). In Klarbring and Strömberg [28] it was further indicated that in large deformation problems, the equivalences in (1) no longer holds, and the appealing properties of $1 / 2 f^{T} u$ as an objective function are lost, while they are mostly kept by the proper potential energy function.

When treating design optimization problems of topology type emanating from other physical domains than linear elasticity, natural analogies of compliance have been used. An almost complete analogy is found in problems based on Poisson's equation, such as heat flow and Darcy flow [19, 10, 14]. In Stokes flow problems compliance type objectives have also been used $[9,18,45,20]$. However, a surprising property of these formulations is that black and white designs appear without the need for penalizations such as that of SIMP. The effect is similar to the self-penalization property reported in, e.g., Wein et al. [44]. An optimization formulation for naturally discrete state problems such as pipe flow has also been formulated using an objective of compliance type [26]. Contrary to truss optimization problems, this formulation does not result in a convex problem. Another area where compliance-like design objectives have been used is that of maximum degradation or damage of a load-carrying structure $[2,3,4]$. However, in these problems the objective is to maximizing compliance instead of minimizing it. It also turns out that, similarly to the self-penalization property of Darcy flow problems, certain parameterizations of damage results in purely black and white solutions. The present paper gives a unified theory that covers all of these design problems from different physical domains and in the process gives a clear explanation for the self-penalization property.

The unified design theory is built on the fact that a large class of linear physical theories is defined by a certain common mathematical structure. This class contains the linear theories of elasticity, viscous fluid flow, thermal flow and electrostatics, to mention the most well known members. Thorough investigations based on this fact can be found in Tonti [38, 39] and Oden and Reddy [31, 32]. For discrete or discretized versions of these theories the duality theory of quadratic programming turns out to contain the indicated mathematical structure. This is seen, e.g., in Strang [42], and for the extended case of contact constraints in Klarbring [24]. Thus, the present paper can be said to extend the general model of physical theories pioneered by Tonti [38, 39] and Oden and Reddy $[31,32]$ to the domain of design problems. In Section 2 we 
give a discrete version of the theory of Tonti and show how the special cases are related to the general case. The extension to design problems is then made in Section 3, while Section 4 presents conclusions.

\section{State problem}

It is natural to divide the presentation into a preliminary section dealing only with the statement of equations of particular physical theories and a core section which deals with the design problems. As described in the introduction there is a common mathematical structure among the linear theories considered and, therefore, we start by introducing the abstract structure, after which we give some particular examples.

\subsection{Master state problem}

The canonical equations of Tonti $[38,39] \mathrm{read}$

$$
A^{T} y=f, \quad y=C e, \quad e=A x-e_{0} .
$$

The first equation is termed the balance equation, the second the constitutive equation and the third the definition equation. We will think of these equations as matrix equations, where $A$ and $C$ are matrices and other symbols indicate vectors. It is also possible to regard these equations in a general operator sense [31, 32]. This will, however, only be briefly used in the special cases in the following. Moreover, it will be assumed that $C$ is a positive definite matrix and $A$ has full column rank. We may call $x$ the nodal vector, $e$ and $y$ the first and second edge vectors, and $f$ and $e_{0}$ the node and edge source vectors. Moreover, we frequently come across a form of these equations where $e$ is eliminated:

$$
A^{T} y=f, \quad C^{-1} y-A x=-e_{0} .
$$

Under the given assumptions, these equations have a unique solution $x$ and $y$, regarding the other vectors and the matrices as given data. These solution vectors may also be characterized as solutions of two optimization problems where the objective functions, or state problem functionals, are as follows:

$$
\Pi(x)=\frac{1}{2}\left(A x-e_{0}\right)^{T} C\left(A x-e_{0}\right)-f^{T} x, \quad \Pi^{*}(y)=\frac{1}{2} y^{T} C^{-1} y+e_{0}^{T} y .
$$

A solution $(x, y)$ of $(3)$ is such that $x$ minimizes $\Pi(x)$, and $y$ minimizes $\Pi^{*}(y)$ under the constraint $A^{T} y=f$. An important property related to these extremum problems is the duality property

$$
\max _{x}\{-\Pi(x)\}=\min _{y}\left\{\Pi^{*}(y): A^{T} y=f\right\} .
$$

The duality theory of quadratic programming, originally given by Dorn [15] and also found in Klarbring [24], contains these results.

The blanket assumption that $C$ is positive definite should be noted. In fact, when structural topology optimization problems are considered by a so-called ground structure approach, there is need to relax this assumption to the case 


\begin{tabular}{l||l|l|l|l|l}
$\begin{array}{l}\text { Master } \\
\text { problem }\end{array}$ & $\begin{array}{l}\text { nodal vec- } \\
\text { tor } x\end{array}$ & $\begin{array}{l}\text { first edge } \\
\text { vector } e\end{array}$ & $\begin{array}{l}\text { second } \\
\text { edge } \\
\text { vector } y\end{array}$ & $\begin{array}{l}\text { node } \\
\text { source } f\end{array}$ & $\begin{array}{l}\text { edge } \\
\text { source } e_{0}\end{array}$ \\
\hline \hline $\begin{array}{l}\text { Linear } \\
\text { elasticity }\end{array}$ & $\begin{array}{l}\text { displace- } \\
\text { ment } \\
u\end{array}$ & strain $\varepsilon$ & stress $\sigma$ & force $f$ & $\begin{array}{l}\text { initial } \\
\text { strain } \\
-\varepsilon_{0}\end{array}$ \\
\hline $\begin{array}{l}\text { Truss } \\
\text { problem }\end{array}$ & $\begin{array}{l}\text { displace- } \\
\text { ment } \\
u\end{array}$ & $\begin{array}{l}\text { bar elon- } \\
\text { gation } e\end{array}$ & bar force $s$ & $\begin{array}{l}\text { nodal } \\
\text { forces } f\end{array}$ & $\begin{array}{l}\text { misfit vec- } \\
\text { tor }-e_{0}\end{array}$ \\
\hline $\begin{array}{l}\text { Darcy- } \\
\text { Stokes } \\
\text { flow }\end{array}$ & pressure $p$ & $\begin{array}{l}\text { effective } \\
\text { force } e\end{array}$ & velocity $v$ & $\begin{array}{l}\text { source of } \\
\text { fluid } s\end{array}$ & $\begin{array}{l}\text { external } \\
\text { force }-g\end{array}$ \\
\hline Pipe flow & pressure $p$ & $\begin{array}{l}\text { pipe force } \\
e\end{array}$ & $\begin{array}{l}\text { volume } \\
\text { flow } q\end{array}$ & $\begin{array}{l}\text { external } \\
\text { inflow } q^{e}\end{array}$ & $\begin{array}{l}\text { external } \\
\text { force }-g\end{array}$
\end{tabular}

Table 1: Correspondence between master problem and special cases.

of a positive semi-definite $C$. This is so since parts of the system (structural members or finite elements) are "removed" by letting certain measures, usually of geometrical nature, such as cross-sectional areas, approach zero. Clearly, $C^{-1}$ does not exist in this case, but by considering non-singular submatrices of $C$, a problem of the same type as $\Pi^{*}(y)$ can nevertheless be formulated, as done for the particular cases of truss structures in Petersson [33], frame structures in Fredricson et al. [17], and for pipe flow in Klarbring et al. [26]. However, for clarity of presentation and ideas we refrain from such a relaxed assumption when presenting the general mathematical theory. Extensions in this direction are discussed in relation to the particular concrete examples.

\subsection{Linear elasticity}

The differential equations of static linear elasticity read

$$
\operatorname{div} \boldsymbol{\sigma}+\boldsymbol{f}=\mathbf{0}, \quad \boldsymbol{\sigma}=\boldsymbol{E} \boldsymbol{\varepsilon}, \quad \boldsymbol{\varepsilon}=\frac{1}{2}\left(\nabla \boldsymbol{u}+(\nabla \boldsymbol{u})^{T}\right) .
$$

Here $\boldsymbol{\sigma}, \boldsymbol{\varepsilon}$ and $\boldsymbol{u}$ are fields of stress, strain and displacement, respectively. Usually these are the unknowns of a problem formulation. Moreover, $\boldsymbol{f}$ and $\boldsymbol{E}$ are external forces and elasticity coefficients, respectively. These are usually regarded as data.

Equation (5) together with appropriate boundary conditions can be seen as a special case of the canonical equations (2) if these are given an operator meaning. Moreover, Besseling [7] showed how to define vectors of generalized stresses and strains (denoted $\sigma$ and $\varepsilon$ ) for displacement finite elements such that a discretized version of linear elasticity becomes

$$
A^{T} \sigma=f, \quad \sigma=C \varepsilon, \quad \varepsilon=A u+\varepsilon_{0} .
$$

For easy comparison with the master problem we have used the same notation for matrices in this particular problem as in (2). The matrix $A$ is defined on purely geometrical grounds and $C$ contains the elasticity coefficients. The force vector $f$ corresponds to the vector with the same name in the master problem, 
while the displacement vector $u$ corresponds to $x$. The vector $-e_{0}$ corresponds to $\varepsilon_{0}$, and is a vector of initial strain which may be produced by non-zero displacements boundary conditions. Table 1 gives the one-to-one correspondences between this problem and the master problem, as well as the correspondence to other problems to be defined below.

\subsection{Truss equations}

A truss consists of bars that connect nodes. A displacement state is associated with these nodes. The mathematical structure given above for a linear elastic continuum discretized by finite elements, is found also for a truss consisting of linear elastic bars under a small displacement assumption, as shown in a large number of places in the literature, e.g., Christensen and Klarbring [11]. Let there be $m$ bars, the directions of which are defined by vectors $\gamma_{i}$ containing direction cosines. For the case of $n$ displacement components, the balance equation and the definition equation is then defined as

$$
\begin{aligned}
& f=\sum_{i=1}^{m} \gamma_{i} s_{i} \Leftrightarrow f=A^{T} s, \\
& e_{i}=\gamma_{i}^{T} u-e_{0 i}, \quad i=1, \ldots, m \quad \Leftrightarrow \quad e=A u-e_{0},
\end{aligned}
$$

where $s=\left(s_{1}, \ldots, s_{m}\right)^{T}$ is a vector of bar forces, $e=\left(e_{1}, \ldots, e_{m}\right)^{T}$ is a vector of bar elongations, $u=\left(u_{1}, \ldots, u_{n}\right)^{T}$ a vector of nodal displacements, $f$ is a vector of nodal forces and $e_{0}=\left(e_{01}, \ldots, e_{0 m}\right)^{T}$ is a vector representing the misfit of bar lengths, producing initial bar elongations. The constitutive equation is

$$
s_{i}=\frac{a_{i}}{\ell_{i}} E e_{i}, \quad i=1, \ldots, m \quad \Leftrightarrow \quad s=C u,
$$

where $C=\operatorname{diag}\left\{a_{i} E / \ell_{i}\right\}$, and $a_{i}, \ell_{i}, i=1, \ldots, m$ are cross-sectional areas and lengths of bars, respectively. The identification of these equations with the master problem is immediate and basically identical with that of linear elasticity in the previous subsection, as shown in Table 1.

Other types of discrete linear elastic structures can also be fitted to the master problem. Closely related to truss structures are frame structures, the stiffness optimization problem of which was treated in Fredricson et al. [17].

\subsection{Darcy-Stokes flow}

Flow of a viscous incompressible fluid, that is slow enough for inertial forces to be neglected, is governed by the following set of differential equations:

$$
\operatorname{div} \boldsymbol{\sigma}+\boldsymbol{f}=\mathbf{0}, \quad \boldsymbol{d}=\frac{1}{2}\left(\nabla \boldsymbol{v}+(\nabla \boldsymbol{v})^{T}\right), \quad \boldsymbol{\sigma}=-p \boldsymbol{I}+\mu \boldsymbol{d}, \quad \operatorname{div} \boldsymbol{v}=s .
$$

Here, $\boldsymbol{\sigma}, \boldsymbol{d}, \boldsymbol{v}$ and $p$ are fields of stress, rate-of-deformation, velocity and pressure, respectively. These are the unknowns of the natural problem formulation. The force field $\boldsymbol{f}$ is taken to be composed of a constant known part $\boldsymbol{g}$ and a drag part that depends linearly on the velocity, i.e., $\boldsymbol{f}=\boldsymbol{g}-\alpha \boldsymbol{v}$. The constants $\mu$ and $\alpha$ are the viscosity and the inverse permeability, respectively. The field $s$ represents a distributed source of fluid. The case when $\alpha$ equals zero is known 
as Stokes flow. When, on the other hand, terms involving $\mu$ can be neglected we have a Darcy flow.

For appropriate boundary conditions, a mixed finite element discretization of equations (10) results in the following matrix equations, see Borrvall and Petersson [9] or Wiker et al. [45]:

$$
C^{-1} v-A p=g, \quad A^{T} v=s,
$$

where $C^{-1}=K^{\alpha}+K^{\mu}$ is the positive definite and symmetric system matrix, consisting of two parts originating from viscosity and inverse permeability, respectively. $A^{T}$ is the matrix version of the divergence operator, meaning that its transpose $A$ can be regarded as a matrix version of the gradient operator with negative sign. The unknown vectors are the velocity vector $v$ and the pressure vector $p$. The vectors $g$ and $s$, representing external force and fluid sources, are given data. The comparison with the master problem becomes complete by introducing the first edge vector $e$, satisfying $e=A p+g$, which may be termed an effective force. Thus, $g$ corresponds to $-e_{0}$ and $s$ to $f$, of the master problem.

Eliminating $v$ from (11) gives

$$
A^{T} C A p=s-A^{T} C g \text {. }
$$

For the case of pure Darcy flow, when $\mu$ is zero, this is usually the equation system directly derived since then $\boldsymbol{v}$ is easily eliminated already at the stage of the continuous system (10). The pure Darcy problem is also completely analogous to the classical heat conduction problem, which is obtained by replacing the permeability by the heat conduction coefficient.

\subsection{Pipe flow}

Similarly to the truss problem, we consider networks consisting of $n$ nodes or junctions and $m$ segments or pipes. Such a problem was discussed in Klarbring et al. [26] and it summarized here. The balance equation is given by Kirchhoff's law, i.e., for each time interval, the fluid volume provided to a node through connecting segments add up to the volume leaving the network at the node. We also define the segment pressure drops $\Delta p_{k}$ in terms of a vector $p=\left(p_{1}, \ldots, p_{n}\right)^{T}$ of nodal pressures. These equations become

$$
\begin{aligned}
& q^{e}=\sum_{i=1}^{m} \gamma_{i} q_{i} \quad \Longleftrightarrow q^{e}=A^{T} q, \\
& \Delta p_{i}=\gamma_{i}^{T} p, i=1, \ldots, m \quad \Longleftrightarrow \Delta p=A p,
\end{aligned}
$$

where $q=\left(q_{1}, \ldots, q_{m}\right)^{T}$ is a vector of volumetric flow rates, $\Delta p$ is a corresponding vector of pressure drops and $q^{e}$ is a vector of external inflow of fluid. The vectors $\gamma_{i}$ contain 1 and -1 in two positions, corresponding to the connected nodes, and 0 in all other positions. The constitutive equation is defined by Hagen-Poiseuille's equation, and reads

$$
q_{k}=\frac{a_{i}^{2}}{8 \pi \mu \ell_{i}}\left(\Delta p_{i}+g_{i} \ell_{i}\right), \quad i=1, \ldots, m \quad \Longleftrightarrow \quad q=C(\Delta p+g),
$$

where $C=(1 / 8 \pi) \operatorname{diag}\left\{a_{i}^{2} / \ell_{i}\right\}$, and, as for trusses, $a_{i}$ and $\ell_{i}$ are cross-section areas and lengths of segments, while $\mu$ is the viscosity. $g_{i}$ are forces per volume 
and form a vector $g$ having elements $g_{i} \ell_{i}$. As for the Darcy-Stokes case the first edge vector $e$ is defined by $e=A p+g$, and termed the effective force.

In Klarbring et al. [26] two types of boundary conditions were defined by dividing the set of nodes into two subsets. One set contains the nodes where volume flows $q_{i}^{e}$ are prescribed quantities and the other set contains the nodes where a pressure $p_{i}$ is prescribed. However, for simplicity we will here assume that the flow is prescribed at all nodes under consideration, i.e., the whole vector $q_{i}^{e}$ is prescribed. Actually this is no restriction since given pressures can be included into the vector $g$ which is also seen as problem data.

\section{Design problems}

We now turn to the design problem. In each of the specific models discussed above we let certain variables, usually of geometrical meaning, be unknown and subject to the solution of an optimization problem. It turns out that it is possible to extend the master state problem, i.e., the canonical equations (2), such that master design formulations, that contains the specific cases, are obtained.

\subsection{Master design problems}

A vector of design variables $\xi$,which belongs to a bounded, closed and convex set $\Delta$, is introduced. The constitutive matrix is taken to depend on the design in a specified manner, i.e., $C=C(\xi)$. It is assumed that $C(\xi)$ is positive definite for all $\xi \in \Delta$. However, as indicated in Section 2.1, an extension to the more general case of a positive semi-definite $C(\xi)$ is of interest and indicated for some particular examples in the following subsections. The objective functions of the dual optimization problems now become functions of the design and we write $\Pi(x, \xi)$ and $\Pi^{*}(y, \xi)$. As an objective for our design optimization we take the value given by (4), i.e., our design objective function is

$$
\phi(\xi)=\max _{x}\{-\Pi(x, \xi)\}=\min _{y}\left\{\Pi^{*}(y, \xi): A^{T} y=f\right\} .
$$

By using (3), it is shown that

$$
\phi(\xi)=\frac{1}{2} e_{0}^{T} y(\xi)+\frac{1}{2} f^{T} x(\xi),
$$

where $y(\xi)$ and $x(\xi)$ are solutions of (3) for a given design $\xi \in \Delta$. This expression for $\phi(\xi)$ is used for interpreting its physical meaning in particular cases. By direct calculations we may also find other expressions for $\phi(\xi)$ :

$$
\begin{aligned}
\phi(\xi)=-\frac{1}{2} y(\xi)^{T} C^{-1} y(\xi) & +f^{T} x(\xi) \\
= & \frac{1}{2}\left(A x(\xi)-e_{0}\right)^{T} C\left(A x(\xi)-e_{0}\right)+e_{0}^{T} y(\xi),
\end{aligned}
$$

which shows that if $f$ is zero, then $\phi(\xi)$ is always non-positive and if $e_{0}$ is zero, then $\phi(\xi)$ is always non-negative.

Since both of the design goals of maximizing or minimizing $\phi(\xi)$ can be of interest in different situations we investigate two different design problems:

$$
\left(\mathbb{P}_{\min -\phi}\right) \quad \min _{\xi \in \Delta} \phi(\xi) \quad \text { and } \quad\left(\mathbb{P}_{\max -\phi}\right) \quad \max _{\xi \in \Delta} \phi(\xi) .
$$


By using the two expressions for $\phi(\xi)$ in (16) we can extract some different properties of these problems and their solutions. We chose to separate two classes of problems, treated in two different subsections:

\subsubsection{Saddle point problems}

Substituting the max-expression of $(16)$ into $\left(\mathbb{P}_{\text {min- } \phi}\right)$ gives

$$
\min _{\xi \in \Delta} \max _{x}\{-\Pi(x, \xi)\}=-\max _{\xi \in \Delta} \min _{x} \Pi(x, \xi),
$$

which suggests the saddle point problem

$$
\left(\mathbb{P}_{\text {min- } \phi}^{\text {saddle }}\right) \quad\left\{\begin{array}{l}
\text { Find } x^{*} \text { and } \xi^{*} \in \Delta \text { such that } \\
\Pi\left(x^{*}, \xi\right) \leq \Pi\left(x^{*}, \xi^{*}\right) \leq \Pi\left(x, \xi^{*}\right) \\
\text { for all } x \text { and } \xi^{*} \in \Delta .
\end{array}\right.
$$

If $(x, \xi)$ solves $\left(\mathbb{P}_{\text {min- } \phi}^{\text {saddle }}\right)$, then the $\xi$-part also solves $\left(\mathbb{P}_{\text {min- } \phi}\right)$. However, the opposite is not generally true unless $\Pi(x, \xi)$ is a convex-concave function. Nevertheless, since $\Pi(x, \xi)$ is always a convex function of $x$, if $\Pi(x, \xi)$ is also a concave function of $\xi$, then a solution of $\left(\mathbb{P}_{\min -\phi}^{\text {saddle }}\right)$ is known to exist, and $\left(\mathbb{P}_{\text {min- } \phi}\right)$ and $\left(\mathbb{P}_{\text {min- } \phi}^{\text {saddle }}\right)$ are essentially equivalent. These results can be found in Ekeland and Temam [16] and were reported in the frame of structural stiffness optimization in Petersson and Klarbring [34].

Another saddle point problem is suggested by substituting the min-expression of (16) into $\left(\mathbb{P}_{\max -\phi}\right)$ :

$$
\left(\mathbb{P}_{\max -\phi}^{\text {saddle }}\right) \quad\left\{\begin{array}{l}
\text { Find } y^{*} \in Y \text { and } \xi^{*} \in \Delta \text { such that } \\
\Pi^{*}\left(y^{*}, \xi\right) \leq \Pi^{*}\left(y^{*}, \xi^{*}\right) \leq \Pi^{*}\left(y, \xi^{*}\right) \\
\text { for all } y \in Y \text { and } \xi^{*} \in \Delta
\end{array}\right.
$$

where $Y=\left\{y: A^{T}=f\right\}$. Similarly to the first saddle point problem, if $(y, \xi)$ solves $\left(\mathbb{P}_{\max -\phi}^{\text {maddle }}\right)$, then the $\xi$-part also solves $\left(\mathbb{P}_{\max -\phi}\right)$. If $\Pi^{*}(y, \xi)$ is a concave function of $\xi$, a solution of $\left(\mathbb{P}_{\max -\phi}^{\operatorname{maddle}}\right)$ is known to exist and the two max-type problems are equivalent. A special and usual situation when concavity in the $\xi$ variable holds, implying that solutions exist for the corresponding saddle point problems, is when $C(\xi)$ or $C(\xi)^{-1}$ is a linear function of $\xi$.

\subsubsection{Extreme point solutions}

While the above saddle point problems are of definite interest in some branches of design optimization, see, e.g., Petersson and Klarbring [34], they are of limited interest in topology optimization, where, on the other hand, the following results are central:

If $\Pi^{*}(y, \xi)$ is concave in $\xi$, then $\phi(\xi)$ is concave and $\left(\mathbb{P}_{\text {min- } \phi}\right)$ has a solution which is an extreme point of $\Delta$.

and similarly

If $-\Pi(x, \xi)$ is convex in $\xi$, then $\phi(\xi)$ is convex and $\left(\mathbb{P}_{\max -\phi}\right)$ has a solution which is an extreme point of $\Delta$. 
These results follow from the following facts:

1. Functions that are pointwise minima of a set of concave function are concave, and functions that are pointwise maxima of a set convex functions are convex [37].

2. A convex function, finite on all of space, is continuous [37].

3. By Bolzano-Weierstrass theorem, a continuous function on a compact set attains its maximum and minimum on that set [13].

4. If a convex function attains its maximum on a convex and compact set, then it is attained at an extreme point of that set [29].

Thus, item 1 shows that $\phi(\xi)$ is convex or concave under the respective assumption. Items 2 and 3 show that $\left(\mathbb{P}_{\min -\phi}\right)$ and $\left(\mathbb{P}_{\max -\phi}\right)$ are solvable; and, finally, item 4 shows that solutions that are extreme points exist.

As will be clear from the special cases, extreme points will be related to so-called black and white designs in topology optimization. This is particularly well shown in the special case studied by Stolpe and Svanberg [41]. They show that if the convex and compact set is

$$
\Delta=\left\{\xi \in[0,1]^{n}: \sum_{j=1}^{n} \xi_{j}=M\right\},
$$

and $M$ is a positive integer $(\leq n)$, then every extreme point of $\Delta$ is an integer point. The proof is easily extended to non-integer constants $M$ in the sense that in such cases at most one variable will be non-integer. We call such a solution an almost integer solution.

An important special case of $\xi \mapsto C(\xi)$, that holds for all applications pointed at in this paper, is

$$
C(\xi)=\operatorname{diag}\left\{E\left(\xi_{j}\right) C_{j}\right\} .
$$

That is, the matrix of the constitutive equation is block diagonal consisting of $n$ blocks $E\left(\xi_{j}\right) C_{j}$ (related to segments or elements in the particular applications), where $C_{j}$ is a positive definite element constitutive matrix and $E\left(\xi_{j}\right)$ are scalings of these matrices such that $0<\epsilon \leq E\left(\xi_{j}\right) \leq 1$ for all $\xi \in \Delta$, and $\Delta$ is given by (19). The small number $\epsilon$ is needed to avoid that $C(\xi)$ becomes singular. The inverse of $C(\xi)$ is given by

$$
C(\xi)^{-1}=\operatorname{diag}\left\{h\left(\xi_{j}\right) C_{j}^{-1}\right\}, \quad h\left(\xi_{j}\right)=1 / E\left(\xi_{j}\right) .
$$

Combining this special case with the above conclusions we find that if $E\left(\xi_{j}\right)$ is concave, e.g., linear, then $\left(\mathbb{P}_{\max -\phi}\right)$ has a solution which is an almost integer point of $\Delta$. Similarly, we find that if $h\left(\xi_{j}\right)$ is concave, then $\left(\mathbb{P}_{\min -\phi}\right)$ has a solution which is an almost integer point of $\Delta$. These results are essentially found in the case of linear elasticity in Stolpe and Svanberg [41]. However, at least superficially, the proof of the second statement is different. Concave $E\left(\xi_{j}\right)$ and $h\left(\xi_{j}\right)$ also guarantees validity of saddle point problems and the situation can be summarized in Table 2.

In a large number of applications of topology optimization it is necessary to use regularization of underlying continuum problems. If this is not introduced 


\begin{tabular}{c||c|c|c|c} 
& $\begin{array}{c}\left(\mathbb{P}_{\text {min- } \phi}^{\text {saddle }}\right) \\
=\left(\mathbb{P}_{\text {min- } \phi}\right)\end{array}$ & $\begin{array}{c}\left(\mathbb{P}_{\text {max- }}^{\text {maddle }}\right) \\
=\left(\mathbb{P}_{\text {max- }}\right)\end{array}$ & $\begin{array}{c}\left(\mathbb{P}_{\text {min- } \phi}\right) \\
\text { has extreme } \\
\text { point solution }\end{array}$ & $\begin{array}{c}\left(\mathbb{P}_{\text {max }-\phi}\right) \\
\text { has extreme } \\
\text { point solution }\end{array}$ \\
\hline $\begin{array}{c}E\left(\xi_{j}\right) \\
\text { concave }\end{array}$ & Yes & & & Yes \\
\hline$h\left(\xi_{j}\right)$ & & Yes & Yes & \\
concave & & & &
\end{tabular}

Table 2: Concave functions $E\left(\xi_{j}\right)$ and $h\left(\xi_{j}\right)$ implies properties according to the table. For convex functions conclusions cannot be drawn in a similar way.

anomalies such as checkerboard patterns and mesh dependency occur [35]. The most popular way of regularizing is through the use of filters, which in our discrete setting can be represented by a function $F$ such that

$$
\rho_{j}=F_{j}(\xi)
$$

where $\rho_{j}$ are elements of a vector $\rho$ of filtered design variables. Equations (20) and (21) are now replaced by

$$
C(\xi)=\operatorname{diag}\left\{E\left(F_{j}(\xi)\right) C_{j}\right\}, \quad C(\xi)^{-1}=\operatorname{diag}\left\{h\left(F_{j}(\xi)\right) C_{j}^{-1}\right\} .
$$

We may now use the following fact: If a function $f(u)$ is convex and increasing and a function $u(x)$ is convex, then the composition $f(u(x))$ is convex. The opposite holds for concave and decreasing functions. Since the filters in their standard form are linear and $E$ and $h$ typically are increasing and decreasing, respectively, in the applications, we conclude that linear filters do not change conclusions concerning integer, i.e., black and white, solutions. For non-linear filters, such as those based on image morphology operators [40] and regularized Heaviside step functions [21], properties of convexity and concavity have recently been investigated by Svanberg and Svärd [43], leading to similar conclusions. Note, however, that even if the design variable $\xi$ is of almost integer type, this does not hold for the filtered or physical variable $\rho$. Concerning methods based on element-independent interpolation of density fields, e.g., the pointwise approximation of Luo et al. [30], an investigation of convexity and concavity properties within the present frame is less straightforward since the special form (23) for the matrix $C(\xi)$ does no longer hold.

Another possible regularization method is to use bounds for the design gradient. The $L_{p}$-norm of this gradient introduces a family of regularizing constraints, the extreme cases of which are the perimeter constraint and the slope constraint, see Borrvall [8]. When such constraints are added to $\Delta$, extreme points are no longer black and white. For the slope constraint, the width of transition regions can be estimated, see [8]. For the discretized perimeter constraint Stolpe and Svanberg [41] show, by means of a small example, that extreme points can now contain elements that have the value $1 / 2$, while numerical examples show that in larger problems such intermediate values are rather few. 


\subsection{Linear elasticity}

In the special case of linear elasticity, equation (17) becomes

$$
\phi(\xi)=\frac{1}{2} f^{T} u(\xi)-\frac{1}{2} \varepsilon_{0}^{T} \sigma(\xi) .
$$

The most frequent problem considered in linear elasticity is $\left(\mathbb{P}_{\min -\phi}\right)$ : the standard case of no initial strain is treated in text-books, see $[11,5]$, and the extension to non-zero initial strain was recently discussed in Klarbring and Strömberg [27]. From (18) we conclude that if there is no initial strain present, i.e., $\varepsilon_{0}=0$, then $\phi(\xi) \geq 0$, meaning that $f$ and $u(\xi)$ are at acute angle, and $\left(\mathbb{P}_{\text {min- } \phi}\right)$ is the problem of minimizing the positive projection of $u(\xi)$ on $f$. On the other hand, if there are no external forces, i.e., $f=0$, then (18) implies that $\phi(\xi) \leq 0$ and $\varepsilon_{0}$ and $\sigma(\xi)$ are at acute angle; $\left(\mathbb{P}_{\text {min- } \phi}\right)$ then means maximizing the positive projection of $\sigma(\xi)$ on $\varepsilon_{0}$. Both of these objectives can be interpreted as if we seek a structure which is as stiff as possible. In the general case, when both external load and initial strain are non-zero, $\left(\mathbb{P}_{\text {min- } \phi}\right)$ includes a combination of the two objectives.

For two-dimensional structures, $\xi$ may be connected to the thickness of the structure, i.e., sizing optimization. Then $E\left(\xi_{j}\right)$ will be a linear function of $\xi$ and $\left(\mathbb{P}_{\text {min- } \phi}\right)$ can be represented by the convex saddle point problem $\left(\mathbb{P}_{\min -\phi}^{\text {maddle }}\right)$. In topology optimization, on the other hand, $\xi$ represents a generalized density. We are then interested in achieving black and white designs, which means that we like to have a concave $h\left(\xi_{j}\right)$. Reasoning from a linear $h\left(\xi_{j}\right)$, Stolpe and Svanberg [41] derive a family of interpolation functions $E\left(\xi_{j}\right)$, where a certain parameter is set to produce convex or concave objective functions. This family of functions has been termed the family of RAMP functions. It may also be of interest to note that that the classical SIMP interpolation does not produce concave objective functions but, nevertheless, Stolpe and Svanberg [41] show that there are integer solutions for large enough exponents (see also Rietz [36]). However, it should be noted that a concave objective function is difficult to use in practice. Numerical calculations are therefore usually performed for interpolations that do not satisfy this requirement. Continuation methods where parameter updates are made to approach the concave case may be useful, see section 1.5.4 of Bendsøe and O. Sigmund [5] for a discussion of this.

When $\xi$ represents thicknesses (two-dimensional problem) or generalized densities (three-dimensional problem), a value $\xi_{i}$ such that $E\left(\xi_{i}\right)=0$ means completely removed material, and in a ground structure approach to topology optimization this may result in a singular matrix $C(\xi)$. However, the saddle point theory of Ekeland and Temam [16] can still be used to prove existence of solutions of $\left(\mathbb{P}_{\text {min- } \phi} \phi\right)$, as shown in Petersson and Klarbring [34]. Moreover, the small number $\epsilon$, introduced below equation (20), can also be made to approach zero by perturbing $E\left(\xi_{j}\right)$ or $\Delta$. Intuitively, solutions for the non-singular case should then be approach by solutions for the singular case. That this is indeed the case in a certain sense is shown for some different cases in Achtziger [1], Petersson [33] and Fredricson et al. [17].

The opposite problem, $\left(\mathbb{P}_{\max -\phi}\right)$, has also been considered for linear elasticity structures; namely for the problem of maximum damage or degradation, see [2, $3,4]$. Again $\xi$ is a generalized density. Both the convex problem, corresponding to $\left(\mathbb{P}_{\max -\phi}^{\operatorname{maddle}}\right)$, and the problem corresponding to a concave objective function, 
producing solutions where each element is either undamaged or fully damaged, have been considered. These two problem types are obtained by using linear interpolation for $h\left(\xi_{j}\right)$ or $E\left(\xi_{j}\right)$, respectively. As for the case of $\left(\mathbb{P}_{\text {min- } \phi}\right)$ it is possible to relax the condition of non-singularity of $C(\xi)$, see Achtziger et al. [4] and Achtziger and Bendsøe [2].

\subsection{Truss equations}

For the case of a truss the objective function (17) becomes

$$
\phi(\xi)=\frac{1}{2} f^{T} u(\xi)-\frac{1}{2} e_{0}^{T} s(\xi)
$$

Its interpretation is identical to that of linear elasticity. The most natural choice of $\xi$ is as a linear scaling of the cross-section areas of bars. Problem $\left(\mathbb{P}_{\min -\phi}\right)$ is then represented by the convex saddle point problem $\left(\mathbb{P}_{\text {min- } \phi} \phi\right)$ and problem $\left(\mathbb{P}_{\max -\phi}\right)$ becomes a problem with concave objective function. However, the structure of the stiffness matrix $A^{T} C A$ for a truss can be utilized to rewrite $\left(\mathbb{P}_{\min -\phi}^{\text {saddle }}\right)$ as a linear programming problem, see Ben-Tal and Bendsøe [6], and Klarbring et al. [25]. This can be utilized both for theoretical proofs and numerical algorithms, and it enables treatment of the true topology optimization problem, corresponding to zero lower bound for cross-sectional areas.

\subsection{Darcy-Stokes flow}

The objective function (17) becomes in this case

$$
\phi(\xi)=\frac{1}{2} s^{T} p(\xi)-\frac{1}{2} f^{T} v(\xi) .
$$

A problem corresponding to $\left(\mathbb{P}_{\min -\phi}\right)$ has been treated in a series of publications, starting from Borvall and Petersson [9]. In general, the objective is a combination of minimizing pressure and maximizing velocity, but more precise interpretations can be given. As indicated in Subsection 2.4, in the case of Darcy-Stokes flow, $C^{-1}$ is naturally divided into two parts: one part depends linearly on the inverse permeability $\alpha$ and one part depends linearly on the viscosity $\mu$. For pure Darcy flow, i.e., $\mu=0$, the natural form of the problem is (12), with a system matrix $A^{T} C A$ that depends linearly on permeability. This problem can also be interpreted as one of heat flow, where the heat conduction coefficient plays the role of permeability. The permeability or the heat conduction coefficient corresponds to $E\left(\xi_{j}\right)$ of the master problem. In [19] (heat flow) and [10] (Darcy flow) a SIMP type penalization was for used for these coefficients. The situation is then similar to that of linear elasticity and none of the cases in Table 2 applies. However, again similarly to linear elasticity, substitution of SIMP interpolation for RAMP interpolation (using a high enough coefficient) would give concave $h\left(\xi_{j}\right)$ and a $\left(\mathbb{P}_{\min -\phi}\right)$ problem that has extreme point solutions. Similarly to the situation for linear elasticity, a concave objective function is, however, difficult to use numerically and a continuation procedure may be attempted.

For the case when $\mu \neq 0$ it is natural to keep the form (11) of the problem and a linear interpolation of viscosity or inverse permeability gives a linear $h\left(\xi_{j}\right)$, which means that $\left(\mathbb{P}_{\text {min- } \phi}\right)$ has an extreme point solution, i.e., an black and white designs are achieved. This fact was proved for the continuous underlying 
problem in Borvall and Petersson [9] for interpolation of inverse permeability only and in Wiker et al. [45] for the case of interpolation of both viscosity and inverse permeability.

\subsection{Pipe flow}

The objective function (17) is in this case written as

$$
\phi(\xi)=\frac{1}{2}\left(q^{e}\right)^{T} p(\xi)-\frac{1}{2} g^{T} q(\xi) .
$$

The pipe flow version of problem $\left(\mathbb{P}_{\min -\phi}\right)$ is treated in Klarbring et al. [26]. The interpretation of $\phi(\xi)$ is then to minimize the pressure and maximizing the flow. Other more precise interpretations in special cases are discussed in [26]. The natural choice of $\xi$ is to take it as consisting of the cross-sectional areas $a_{i}$. This means that $E\left(\xi_{j}\right) \sim \xi_{j}^{2}$, which, for positive $\xi_{j}$, implies that both $h\left(\xi_{j}\right)$ or $E\left(\xi_{j}\right)$ are convex. Thus, none of the cases indicated in Table 2 are applicable. So in general we can expect problem $\left(\mathbb{P}_{\min -\phi}\right)$ to be a non-convex problem where solutions are generally not taken at extreme points of the solution set. In Klarbring et al. [26], the convergence to the true topology optimization problem for $\epsilon=0$, corresponding to a singular $C(\xi)$, is treated similarly to the treatment of the frame problem in Fredricson et al. [17].

\section{Conclusions}

The classical maximum stiffness problem of structural optimization has historically been used as an informal guide when formulating design optimization problems in new physical domains. Structural degradation and Darcy-Stokes flow problems can be mentioned as examples of this. However, there has been a lack of understanding of the structure and general properties that is then utilized. The present paper achieves such structure by extending to the domain of design optimization the general model of linear physical theories of Tonti. By using state problem functionals in formulating objective functions, properties of convexity and concavity becomes apparent and we are given concrete guidance to which parameterizations produces a particular property: convex saddle point problems, as well as problem formulations that have the - in topology optimization - desired black-and-white property, are obtained in this way.

\section{Acknowledgment}

This research was supported by the Swedish Foundation for Strategic Research under grant No. AM13-0029 and by the Swedish Research Council under contract Dnr: 621-2012-3117.

\section{References}

[1] W. Achtziger, Multiple-load truss topology and sizing optimization: some properties of minimax compliance, Journal of Optimization Theory and Applications, 98(2), 255-280, 1998. 
[2] W. Achtziger and M.P. Bendsøe, Design for maximal flexibility as a simple computational model of damage, Structural and Multidisciplinary Optimization, 10, 258-268, 1995.

[3] W. Achtziger, M.P. Bendsøe and J.E. Taylor, Bounds on the effect of progressive structural degradation, Journal of Mechanics and Physics of Solids, 46(6), 1055-1087, 1998.

[4] W. Achtziger, M.P. Bendsøe and J.E. Taylor, An optimization problem for predicting the maximal effect of degradation of mechanical structures, SIAM Journal of Optimization, 10(4), 982-998, 2000.

[5] M. Bendsøe and O. Sigmund, Topology Optimization, Theory, Methods and Applications, Springer 2002.

[6] A. Ben-Tal and M. Bendsøe, A new method for optimal truss topology design, SIAM Journal of Optimization, 3(2), 322-358, 1993.

[7] J.F. Besseling, Finite element methods, Trends in Solid mechanics, J.F. Besseling, van der Heijden (eds.), Sijthoff and Noordhoff 1979, 53-57.

[8] T. Borrvall, Topology optimization of elastic continua using restriction, Archives of Computational Methods in Engineering, 8(4), 351-385, 2001.

[9] T. Borrvall and J. Petersson, Topology optimization of fluids in Stokes flow, International Journal for Numerical Methods in Fluids, 41, 77-107, 2003.

[10] T. Borrvall, A. Klarbring, J. Petersson, B. Torstenfelt and M. Karlsson, Topology optimization in fluids, Proceedings of the Fifth World Congress on Computational Mechanics (WCCM V), July 7-12, 2002, Vienna, Austria, H.A. Mang, F.G. Rammerstorfer, J. Eberhardsteiner (Eds.)

[11] P.W. Christensen and A. Klarbring, An Introduction to Structural Optimization, Springer 2009.

[12] F.H. Clarke, Optimization and Nonsmooth Analysis, Wiley, New York 1983.

[13] R.W. Cottle, J-S. Pang and R.E. Stone, The Linear Complementarity Problem, Academic Press, Boston 1992.

[14] A. Donoso and O. Sigmund, Topology optimization of multiple physics problems modelled by Poisson's equation, Latin American Journal of Solids and Structures, 1(2), 169-189, 2004.

[15] W.S. Dorn, Duality in quadratic programming, Quarterly of Applied Mathematics, 18(2), 155-162, 1960.

[16] I. Ekeland, R. Temam, Convex Analysis and Variational Problems, NorthHolland Publ. Company, Amsterdam-Oxford 1976.

[17] H. Fredricson, T. Johansen, A. Klarbring and J. Petersson, Topology optimization of frame structures with flexible joints, Structural and Multidisciplinary Optimization, 25, 199214, 2003. 
[18] A. Gersborg-Hansen, O. Sigmund and R.B. Haber, Topology optimization of channel flow problems, Structural and Multidisciplinary Optimization, 30, 181192, 2005.

[19] A. Gersborg-Hansen, M.P. Bendsøe and O. Sigmund, Topology optimization of heat conduction problems using the finite volume method, Structural and Multidisciplinary Optimization, 31(4), 251-259, 2006.

[20] J.K. Guest and J.H. Prevost, Topology optimization of creeping fluid flows using a DarcyStokes finite element, International Journal for Numerical Methods in Enginering, 66, 461484, 2006.

[21] J.K. Guest, J.H. Prevost and T. Belytschko, Achiving minimim lenght scale in topology optimization using nodal design variables and projection functions, International Journal of Numerical Methods in Engineering, 61(2), 238-254, 2004.

[22] W.S. Hemp, Optimum Structures, Clarendon Press, Oxford 1973.

[23] J.-B. Hiriart-Urrity and C. Lemaréchal, Convex Analysis and Minimization Algorithms I, Springer-Verlag, Berlin 1993

[24] A. Klarbring, Quadratic programs in frictionless contact problems, International Journal of Engineering Science, 24(7), 1207-1217, 1986.

[25] A. Klarbring, J. Petersson and M. Rönnqvist, Truss Topology Optimization Involving Unilateral Contact, Journal of Optimization Theory and Applications, 87(1), 1-31, 1995.

[26] A. Klarbring, J. Petersson, B. Torstenfelt and M. Karlsson, Topology optimization of flow networks, Computer Methods in Applied Mechanics and Engineering, 192, 3909-3932, 2003.

[27] A. Klarbring and N. Strömberg, A note on the min-max formulation of stiffness optimization including non-zero prescribed displacements, Structural and Multidisciplinary Optimization, 45(1), 147-149, 2012.

[28] A. Klarbring and N. Strömberg Topology optimization of hyperelastic bodies including non-zero prescribed displacements, Structural and Multidisciplinary Optimization, 47(1), 37-48, 2013.

[29] D.G. Luenberger, Introduction to Linear and Nonlinear Programming, Addison-Wesley, Massachusetts 1973.

[30] Z. Luo, N. Zhang, Y. Wang and W. Gao, Topology optimization of structures using meshless density variable approximants, International Journal for Numerical Methods in Engineering, 93, 443464, 2013.

[31] J.T. Oden and J.N. Reddy, On dual-complementary variational principles in mathematical physics, International Journal of Engineering Science, 12, 1-29, 1974.

[32] J.T. Oden and J.N. Reddy, Variational methods in theoretical mechanics, Springer-verlag, Berlin 1983. 
[33] J. Petersson, On continuity of the design-to-state mapping for trusses with variable topology, International Journal of Engineering Science, 39, 11191141, 2001.

[34] J. Petersson and A. Klarbring, Saddle point approach to stiffness optimization of discrete structures including unilateral contact, Control 85 Cybernetics, 23(3), 461-479, 1994.

[35] J. Petersson and O. Sigmund, Numerical instabilities in topology optimization: A survey on procedures dealing with checkerboards, meshdependencies and local minima, Structural Optimization, 16, 68-75, 1998.

[36] A. Rietz, Sufficiency of a finite exponent in SIMP (power law) methods, Structural and Multidisciplinary Optimization, 21, 159-163, 2001.

[37] R.T. Rockafellar, Convex Analysis, Princeton University Press, Princeton 1970.

[38] E. Tonti, On the mathematical structure of a large class of physical theories, Academia Nationale Dei Lincei, Serie VIII, Vol LII, fasc. 1, Genaio 1972, pp 48-56.

[39] E. Tonti, The reason for analogies between physical theories, Appl. Math. Modelling, 1, 37-50, 1976.

[40] O. Sigmund, Morphology-based black and white filters for topology optimization, Structural and Multidisciplinary Optimization, 33, 401-424, 2007.

[41] M. Stolpe and K. Svanberg, An alternative interpolation scheme for minimum compliance topology optimization, Structural and Multidisciplinary Optimization, 22, 116-124, 2001.

[42] G. Strang, Introduction to Applied Mathematics, Wellesley-Cambridge Press, Massachusetts 1986.

[43] K. Svanberg and H. Svärd, Density filters for topology optimization based on the Pythagorean means, Structural and Multidisciplinary Optimization, 48(5), 859-875, 2013.

[44] F. Wein, M. Kaltenbacher, B. Kaltenbacher, G. Leugering, E. Bänsch and F. Schury, On the effect of self-penalization of piezoelectric composites in topology optimization, Structural and Multidisciplinary Optimization, 43(3), 405-417, 2010.

[45] N. Wiker, A. Klarbring, and T. Borrvall, Topology optimization of regions of Darcy and Stokes flow. International Journal for Numerical Methods in Engineering, 69(7):1374-1404, 2007. 